\title{
Prensa y modernización en América Latina y Chile en la segunda mitad del siglo XIX: la crónica y los cronistas
}

\author{
Eduardo SANTA CRUZ ACHURRA \\ Universidad de Chile \\ esantacr@uchile.cl
}

Recibido: $21 / 02 / 2011$

Aceptado: 19/09/2011

\begin{abstract}
Resumen
En las últimas décadas del siglo XIX se produce un cambio fundamental en las formas de hacer periodismo. El "modelo informativo", como tendencia universal, dejaba atrás a la "prensa doctrinaria", predominante desde la Independencia, en América Latina y Chile, asociado al nacimiento delmercado informativo y de los géneros periodísticos modernos. El naciente campo periodístico se relacionó con el proceso de autonomización del campo intelectual en general, lo que provocó la presencia de un nuevo tipo de escritor que encontró en la prensa y en la crónica un espacio de desarrollo, no exento de tensiones y complejidades. Se trata de describir y problematizar estos procesos como momentos fundantes y que determinaron la evolución de la prensa latinoamericana y chilena hasta la actualidad.

Palabras clave: Prensa Latinoamericana, historia de la prensa chilena, prensa liberal moderna, crónica periodística.

\section{Press and modernization in Latin America and Chile in the second half of the XIX century: the chronicle and chronists}

\begin{abstract}
During the last decades of ninth century there is a change in the way of making journalism. The "information model", as a universal tendency, leaves behind the "doctrinary press" that was predominant since the independence in Chile and Latin América, associated to the birth of the information market and the modern journalism genres. The rising journalism field was related with the automation of the intellectual field in general, that generate the presence of a new kind of writer that found in the press as well as in the chronicle a space of development, not exent of tensions and complexity. The objective is to describe and discuss this process as foundation moments that determined the evolution of the Chilean and latinamerican press till now.
\end{abstract}

Keywords: Latinamerican and Chilean Press, History-Liberal Modern, Chronicle

${ }^{1}$ Proyecto Fondo Nacional de Ciencia y Tecnología № 1085029, Chile: Espacio público y subjetividad en América Latina: Bello, Darío y Martí. El presente texto se inscribe al interior de una investigación más amplia y de mayor alcance que se planteó examinar dos dispositivos culturales propios del siglo XIX en América Latina. En primer lugar, la dimensión proyectual o fundacional que caracteriza la obra de Andrés Bello y, en segundo lugar, las tensiones o desencuentros que, en relación con el mundo moderno, caracteriza obras como los trabajos periodísticos de José Martí, donde interesa ver el modo cómo la modernidad deja de ser primariamente "proyecto" para devenir sobre todo "experiencia". Forma parte constituyente de la descripción de estos dos emplazamientos su análisis en el interior de las transformaciones que vive la prensa en la época y cómo se articulan ellas con el desarrollo del espacio público. En esta investigación el autor de este artículo se desempeñó como coinvestigador. 


\section{Referencia normalizada}

SANTA CRUZ ACHURRA, Eduardo (2011): "Prensa y modernización en América Latina y Chile en la segunda mitad del siglo XIX: la crónica y los cronistas”. Estudios sobre el mensaje periodístico. Vol. 17, núm. 2, págs.: 647-660. Madrid, Servicio de Publicaciones de la Universidad Complutense.

Sumario: 1. Introducción. 2. El periodismo liberal moderno como tendencia universalista y modernizante. 3. Las transformaciones de la prensa chilena. 4. Géneros periodísticos y prensa liberal moderna. 5. Conclusiones. 6. Referencias bibliográficas.

\section{Introducción}

En la segunda mitad del siglo XIX, la prensa latinoamericana vio aparecer las primeras manifestaciones de un nuevo modelo periodístico que desplazó a la prensa doctrinaria predominante durante la mayor parte de la centuria, desde el proceso independentista en adelante (SANTA CRUZ, 2009). Esta tendencia universal se manifestó en la prensa de América Latina, con mayor fuerza y prontitud en unos países que en otros, lo que estaba generalmente determinado por los particulares contextos de cada uno de ellos. Ello significó que la aparición de la prensa informativa y de empresa se expresara como una tendencia que se desarrolló a lo largo de varias décadas y, por tanto, el campo periodístico, por ella desarrollado, asumió, en general, un perfil complejo y heterogéneo donde confluyeron diversos modelos de prensa y en el que la prensa doctrinaria predominante se mantuvo presente incluso hasta principios del siglo XX, en algunos lugares, mientras paulatinamente iba dejando su lugar a la prensa liberal informativa moderna.

\section{El periodismo liberal moderno como tendencia universalista y modernizadora} En EE.UU., primero, ya desde los años '30 habían aparecido periódicos como The Sun (1833) de Benjamin Day o el Morning Herald (1835) de James Gordon Bennett que introdujeron la idea de información sobre hechos espectaculares o novedosos por sobre la difusión de doctrinas o los análisis ideológicos: "fue el Herald quien introdujo sistemáticamente la crónica directa, el embrión del "reportaje" en los diarios" (VÁzQUEZ Montalbán, 1985:162). Poco tiempo después el fenómeno comenzó a manifestarse en Europa, donde Inglaterra había desarrollado ya desde elsiglo XVIII, un tipo de prensa informativa dirigida hacia un mercado en expansión, por oposición a la prensa generalmente controlada por los estados que había prevalecido en la Europa continental durante la primera mitad del siglo XIX: "así es como entre 1830 y 1860 se reúnen los elementos de una transformación completa de la prensa periódica. Los periódicos ya no están reservados a una clase poca numerosa de lectores censatarios; quieren dirigirse a todos los que saben leer y el número de estos se va agrandando [...] El anuncio ya constituye un recurso precioso, aunque todavía no alcanza todo su desarrollo. El periódico a diez céntimos, a un centavo, a un penique anuncia una época nueva" (WeILL, 1994: 152-153).

A la creciente configuración de un público lector masivo se unió un conjunto de innovaciones tecnológicas que se sucedieron unas a otras, tales como la imprenta a vapor, el telégrafo, los cables submarinos, la linotipia y, a finales de siglo, el teléfono y el uso de la electricidad, los que ligados a los nuevos medios de transporte como la 
navegación a vapor y el ferrocarril, crearon las condiciones para que emergiera una nueva forma de hacer periodismo dedicada ahora a "recoger las noticias, aumentar el número de páginas, hacer frente a las mayores tiradas. No se limitó a estas transformaciones materiales; fue necesario adaptar la prensa a las necesidades y a los gustos del público" (WeILL, 1994: 173).

En Francia e Inglaterra aparecieron las agencias de noticias (Havas y Reuter, respectivamente), las que en 1882 comenzaron a transmitir noticias a países latinoamericanos. Por otra parte, comenzó el uso de la ilustración y la fotografía y también apareció paulatina, pero crecientemente el aviso publicitario, el que, en un sistema de prensa de mercado, constituye el mecanismo fundamental para la permanencia y éxito del negocio. Cabe agregar que esta transformación de la prensa dice estrecha relación con las transformaciones económicas, sociales, políticas y culturales queconfiguraban una nueva etapa de la modernización capitalista, ya que se trata de una condición política e ideológica, sólo posible al consolidarse una hegemonía: "solo cuando el empresario de prensa demostró que necesitaba cierta independencia para garantizar su negocio, y que esa independencia no le enfrentaba necesariamente a un estado y a un orden con los que se sentía identificado, se consumó la victoria de la teoría liberal de la comunicación. Se consuma en el momento en que ya no es un factor de cambio histórico, sino de consolidación del estado burgués" (VÁZqueZ Montalbán, 1985:159).

En América Latina, uno de los casos más significativos en el surgimiento de este nuevo tipo de periódicos es el de La Nación, de Buenos Aires, propiedad de Bartolomé Mitre desde 1870, cuando adquirió la anterior La Nación Argentina, que había sido fundada en 1862. Mitre reestructuró al medio como sociedad anónima y este "desde sus inicios contó con una red de corresponsales propios y de agencias en todo el país y en el exterior. Su planta de personal superó las 4.000 personas. Mitre también se preocupó de dotar al diario con modernas imprentas, que le permitieron lanzar tiradas muy voluminosas para la época" (BARRERA, 2004:158). El caso argentino, en todo caso, no se redujo al diario citado. Por el contrario, más bien éste fue posible gracias a la consolidación de una cierta estabilidad institucional, no exenta de conflictos y turbulencias claro está, y sobre todo de la creciente hegemonía liberal, desde la caída de Rosas, lo que facilitó, entre otras condiciones, el crecimientodel campo periodístico. Al respecto, hacia 1872 ya existían 26 imprentas y 94 periódicos en Buenos Aires (Állvarez y MarTínez, 1992).

Al otro del Río de la Plata, en Uruguay, ocurrió algo similar. Hacia 1886 había, según los mismos autores, 21 diarios y 40 publicaciones periódicas, con unos 30.000 ejemplares de tirada y el caudillo liberal José Battle y Ordoñez fundó ese mismo año, El Día, que si bien fue clausurado el año siguiente, reaparecido en 1889: "convertido en un prototipo de diario de masas: supresión del sistema de suscriptores (característico de la prensa de elite), reducción drástica del precio del ejemplar [...] En 1899, Battle decidió eliminar su carácter político abriendo sus páginas a todo tipo de colaboraciones. Simultáneamente cambió su imagen al ser confeccionado en una nueva rotativa, única en su género en el país" (Álvarez y MARTíneZ, 1992: 160).

Por su lado, en Brasil es posible consignar dos experiencias en la dirección señalada. Por una parte, O Estado de Sao Paulo, fundado en 1875 y Jornal do Commer- 
cio, de Río de Janeiro, periódico fundado en 1827 por el francés Pierre Plancher. En 1890, “asumió la dirección el periodista brasileño José Carlos Rodríguez que lo dotó de una moderna organización y maquinaria" (BARRERA, 2004:163)

Un caso particular lo constituyó México, debido a la presencia y la acción de la dictadura de Porfirio Díaz. Uno de los periódicos más importantes del período fue " $E l$ Siglo XIX", entendido como "el primer intento de hacer un diario moderno, contando para ello con una rotativa importada de Estados Unidos y desplegando una organización interna muy avanzada para la época" (Álvarez y MARTínez, 1992: 86). Dicho medio que reivindicaba "una actitud crítica ante cualquier tipo de gobierno", según los mismos autores, no pudo resistir los embates y restricciones de la dictadura de Díaz, sobre todo a partir de 1885. Sin embargo, lo distintivo del caso mexicano es que la dictadura promovió por su lado la aparición de diarios "modernos". Por ello, los autores citados afirman que "la novedad de este período fue la concepción de la prensa como un negocio, la búsqueda de una mayor difusión y mejor distribución y el descenso de los costes gracias a la introducción de innovaciones tecnológicas". Surgieron los característicos "diarios de a centavo", entre los que fue pionero El Monitor del Pueblo, publicado entre 1885 y 1893" (ÁlvAREZ y MARTínEZ, 1992:121). Junto al anterior, se debe mencionar a El Imparcial, editado desde 1896 por Rafael Reyes Espínola, quien utilizó las primeras linotipias y rotativas que llegaron a México y obtuvo un gran éxito. Lo peculiar es que todas estas experiencias tuvieron respaldo económico y político de la dictadura de Díaz, "la gestión del gobierno fue determinante en la evolución de este tipo de periodismo. En 1888 subvencionaba tres periódicos de la capital [...] y además sostenía al menos 27 periódicos en el resto de los estados" (ÁlvarEZ y MARTíneZ, 1992: 121).

Otro caso a destacar es el de El Comercio, de Lima, fundado en 1839, el que habría sido concebido desde un principio "como un negocio y como tal se explotó [...] Por otro lado, los propietarios tuvieron negocios paralelos y eso permitió que el periódico sobreviviera a acontecimientos y cambios políticos" (ÁLVAREZ y MARTínEZ, 1992: 140). Sin embargo, dichos autores llaman la atención al hecho de que el desarrollo y la difusión del periodismo peruano se circunscribía fundamentalmente a la capital, la que de todas formas "era vista por algunos como 'una ciudad de lectores" " (AGUIRRE, 2004: 276). Distinta fue la situación en otros lugares de la región donde la aparición del periodismo liberal moderno había de esperar hasta la llegada del siglo XX. En Bolivia, por ejemplo, "hasta finales de siglo, la prensa fue medio de expresión de facciones y caudillos" (ÁLVAREZ y MARTíneZ, 1992: 147).

Contradictorio resulta ser el panorama que ofrece la evolución de la prensa en Colombia. Por su lado, Álvarez y MARTínEZ señalan que, ya en 1855, los hermanos Echeverría "confeccionaron el primer periódico de gran formato en el país, El Tiempo. Su intención era producir un medio de expresión política y un órgano literario y de información miscelánea al estilo de los grandes modelos europeos" (ÁLVAREZ y MARTíNEZ, 1992: 135). Dicho medio se publicó hasta 1872. No obstante, otros estudios más bien ponen de relieve lo insuficiente del desarrollo del campo en la segunda mitad del siglo XIX. Así, Eduardo PosADA (2004: 187) afirma que "los colombianos tendrían que esperar hasta el siglo XX para experimentar una prensa de masas". Si bien reco- 
noce el éxito de El Diario de Cundinamarca, lo atribuye al apoyo financiero de los gobiernos de la época, agregando que "en las circunstancias de pobreza de la Colombia decimonónica, los altos costos de producción, las dificultades de circulación por los problemas de transporte y la escasez de lectores parecen obvias explicaciones para la lenta modernización del periodismo" (PosADA, 2004:191). De todos modos, ya hacia la última década del siglo se constata en Colombia la aparición de nuevos medios en distintas ciudades del país, tales como La Consigna (Medellín), La Voz de Antioquia (Medellín), El Impulso (Bucaramanga), La República (Bucaramanga), El Trabajo (Popayán), etc. Los de mayor permanencia fueron Las Noticias, desde 1884 a 1890 y El Correo Nacional, "que llegó a ocupar el primer puesto de la prensa nacional e inició la costumbre de publicar entrevistas" (ÁlVAREZ y MARTínEZ, 1992: 137).

Finalmente, cabe mencionar el caso de Venezuela donde predominaron hasta finales del siglo periódicos doctrinarios y políticos, "era habitual que se multiplicaran en vísperas de elecciones y casi desaparecieran cuando se hacían incómodos al poder. Predominaban las largas disquisiciones doctrinales y los panegíricos o críticas a las personalidades públicas" (ÁLVAREZ y MARTíNEZ, 1992:127). No obstante, en las dos últimas décadas del siglo XIX aparecieron una serie de revistas especializadas temáticamente. Entre ellas, es posible mencionar a La Caricatura editada en 1886 y de corte satírico-humorística; El Cojo Ilustrado, editada en 1892 de corte modernista y positivista; Cosmópolis, editada en 1893 y cuyo contenido era de carácter estético y artístico promoviendo las últimas tendencias en el campo; Gaceta Médica, editada en 1893 y centrada en contenidos relacionados con la Medicina, todas ellas publicadas en Caracas, aunque también hubo otras en diversas ciudades, como El Zulia Ilustrado, de Maracaibo, de 1888. En ese marco, la que adquirió posteriormente mayor resonancia fue la Revista Venezolana, fundada por José Martí, "aunque su negativa a hacer propaganda gubernamental determinó su suspensión y la salida de Mart'”" (ÁlvareZ y MARTíNEZ, 1992: 130).

\section{Las transformaciones de la prensa chilena}

En el caso de la prensa chilena, el nuevo marco legislativo instalado a partir de la Ley de Imprenta promulgada en 1872 creó las condiciones de legitimidad institucional necesarias para que paulatinamente se instalaran proyectos periodísticos que apuntaban hacia la idea de una prensa de empresa. En estas décadas finales del siglo XIX comienza a configurarse el aludido periodismo liberal moderno, que tiene como expresión orgánica la empresa periodística, ya que "cada día gana más terreno la información sobre los comentarios y las polémicas de carácter meramente doctrinario" (VALDEBENITO, 1956:69).

La modernización en el ámbito de las comunicaciones tiene como un componente importante lo que llamamos la diversificación de medios. Ello expresa la articulación de dos fenómenos interconectados y que se retroalimentan mutuamente: el desarrollo de las tecnologías comunicacionales que van permitiendo masificar reproductivamente el uso de variados códigos y formatos y el desarrollo creciente de públicos con algún grado de especialización en sus demandas e intereses culturales. En nuestro país el primer paso en esa dirección se dio en la segunda mitad del XIX, no sólo por el aumento 
en el número de diarios y revistas a lo largo de todo el territorio, sino -más importanteen su carácter. Es el período además en que se va consolidando el lugar de El Ferrocarril, como principal diario capitalino y nacional. Es posible encontrar desde mediados de la década de los ' 60 en adelante, prensa literaria, satírica, embrionariamente informativa y comercial, prensa más o menos vocera de los grupos políticos, etc.

Junto a ello, se va configurando lo que hemos llamado una "esfera pública plebeya", constituyéndose con la aparición de la prensa obrera y de artesanos, ligada a la construcción y posicionamiento de nuevas identidades y actores sociales y de sus organizaciones. Publicaciones como El Duende (1845) y El Pueblo (1846) del tipógrafo Santiago Ramos, pueden ser citadas "...como una de las primeras voces significativas, aunque todavía marginales y poco consistentes, del 'bajo pueblo' urbano" (OSSANDÓn, 1998: 42). Asimismo, es posible mencionar las experiencias de El Precursor (1882) y La Razón (1884-1885), pero también cabe agregar a la Lira y Poesía popular, si bien no reductible al ámbito de la prensa, aunque cumpliera funciones informativas y articulara lo oral y lo escrito, como adecuación a las características de su público (URIBE, 1973).

Se trataría, entonces, de una prensa que va tomando lentamente una cierta "conciencia de si" y de su capacidad de construir, vehicular y difundir sentidos, en definitiva de lograr autonomizarse, en alguna medida, del poder político, cuestión que hacia fines del diecinueve es un proceso central en la constitución de un campo intelectual plenamente moderno. Hay que agregar el caso de la llamada prensa mercantil, orientada fundamentalmente a la información económica. De esta última surgió el único periódico de la época que trascendió hasta la actualidad: El Mercurio de Valparaíso. Fundado el 12 de Septiembre de 1827, tuvo una especie de antecesor en El Telégrafo Mercantil y Político, que circuló entre 1826 y 1827, fundado por Pedro Félix Vicuña. El Mercurio de Valparaíso logró consolidar un nivel de venta que otros periódicos de la época nunca lograron. Instalado al centro de la actividad económica de lo que era el puerto más importante del Pacífico Sur, circulaba por toda su costa: "El único de esos periódicos que había podido subsistir sin la subvención gubernativa, era El Mercurio de Valparaíso, que tenía gran circulación en toda la costa del Pacífico, con muchos avisos y que era, además, entonces el más noticioso y el mejor servido de todos los órganos de la prensa chilena" (BARRos AranA, 2003: 174). En la misma dirección, agrega el citado autor que era "el diario más acreditado y de mayor circulación".

En todo caso, el diario más importante de la época fue El Ferrocarril (OSSANDÓN y SANTA CRUZ, 2001: 44-77), donde vemos aparecer un intento de posicionamiento en la sociedad, a partir de tomar una cierta distancia con el acontecer político inmediato y más bien concentrado en generar debates sobre temas y problemas relacionados con la construcción de un país moderno. En ese sentido, despunta efectivamente, en embrión todavía, un tipo de prensa distinto, que se coloca a medio camino entre el Estado y una opinión pública interesada en formar, ya no tribuna simplemente para la propagación de un ideario que tiene su origen en otro lugar de la sociedad. En ese sentido en El Ferrocarril encontramos ciertos atributos que caracterizarían la emergencia hacia 1860 de una "prensa raciocinante" (OSSANDÓN, 1998), en la cual es posible distinguir tres elementos constitutivos en lo medular: en primer término, 
instalándose en un espacio social que es a la vez origen y destino, que proviene de la separación entre la esfera estatal y la sociedad, reconociendo su lugar en el desarrollo autónomo de esta última. En esa dirección, había que hacer algo más que "distribuir" cultura al modo de los periódicos iluministas. Era preciso ahora "opinar", al decir del mismo autor. Una segunda cuestión dice relación con la distancia que se toma de la prensa doctrinaria y polémica, predominante en la primera mitad del siglo XIX. Más que ser tribuna y barricada, el diario postula como su misión promover la "discusión". Finalmente, un tercer elemento dice relación con la "razón" (o la "lógica", según palabras del diario). Se trataría de instalar un dispositivo en que la razón y la lógica son consideradas como operaciones que a través del raciocinio y argumentación, pueden llegar a ciertas conclusiones.

Un caso distinto, pero igualmente significativo para ilustrar la tendencia que se estaba imponiendo lo constituyó el de $L a$ Tarde. Se trató de un vespertino que comenzó a aparecer el $1^{\circ}$ de Enero de 1897, fundado por los hermanos Galo y Alfredo Irarrázaval Zañartu, y al decir de SiLva CASTRO (1958: 315): "Era su intención hacer un nuevo tipo de periodismo, en que la ligereza de la redacción y la gracia de la forma pudieran combinarse con altivas campañas de orden político". Según el testimonio de uno de sus redactores, La Tarde era: “...nuevo como estilo, sin 'remitidos' ni editoriales de levita y sombrero de ocho luces y, en cambio, lleno de artículos, crónicas, reportajes e instantáneas, ágiles, livianos e informaciones en que los personajes aparecían tal cual, es decir, vivos, hablando, charlando [...] La Tarde era, pues, una atrevida innovación que, a pesar de lo limitado de sus recursos venía a transformar el periodismo de que había sido modelo o patrón el ahuesado Ferrocarril (RodRíGuez MENDOZA, 1955: 68).

Otro caso especial es el de La Epoca, (1881-1892). Como señala OsSANDÓn (1998: $105)$ en su obra citada: "es uno de los diarios que mejor cristaliza los elementos característicos de la prensa moderna: órgano privado (propiedad del empresario Agustín Edwards Ross), independiente del ámbito político estatal, interesado en mantener vínculos con el gobierno [...], con la sociedad civil y con las demandas de distinta naturaleza que provienen de esta esfera y de contribuir a la ampliación de un espacio público, "libre", mediador, informadoraciocinante".

Este periódico fue donde mejor se concentró y se expresó la llamada sensibilidad modernista. Su propio local se constituyó en uno de los lugares físicos donde se reunían un grupo de jóvenes literatos e intelectuales encabezados, nada menos que por Rubén Darío. Sin embargo, los casos anteriores más bien constituyeron experiencias híbridas que quedaron a medio camino, sin instalarse plenamente a la cabeza de la tendencia modernizante. La culminación del proceso está en la fundación de El Mercurio, de Santiago, el $1^{\circ}$ de Junio de 1900: “...Las innovaciones técnicas introducidas por Agustín Edwards, que dieron al público la impresión de que por primera vez leía un diario que fuera capaz de romper viejos moldes, fueron uno de los secretos de su éxito. Equipado con máquinas modernas, enriquecidas sus páginas con amplios servicios de informaciones nacionales y del extranjero y con las colaboraciones de las mejores plumas, pronto se convirtió en el principal diario del país" (VALDEBENITO, 1956: 71).

La aparición de este diario, ya con características plenas de empresa periodística, significó una completa reestructuración de la prensa nacional. Por un lado, surgieron 
otros diarios competidores en la misma perspectiva, como es el caso de El Diario Ilustrado, que introdujo el uso del fotograbado, permitiendo por primera vez la publicación cotidiana de fotografías y la desaparición progresiva de la mayor parte de la antigua prensa capitalina. Lo que ocurrió es que al comenzar el siglo XX se consolidó también la existencia de un mercado informativo, con sus exigencias de competitividad, lo cual significó que: "Económicamente ya no eran posibles los diarios 'pobres'. El sólo subsistir implicaba la necesidad de un fuerte respaldo monetario o capital-dinero o bien (y venía a ser idéntico) circulación y avisaje, éste y aquella íntimamente relacionados" (VIAL CORREA, 1986: 276).

Por último, El Mercurio, de Santiago, fue decisivo en la consolidación definitiva del proceso a través del cual la producción del discurso informativo dirigido a conformar y orientar la discusión pública, quedó entregada a un profesional específico, funcionario de la empresa periodística. Junto a estos profesionales, la prensa liberal moderna contó con la colaboración de los más importantes exponentes de la literatura e intelectualidad nacionales, muchos de ellos de origen provinciano y, en general, de los sectores medios ilustrados emergentes. La estructura formal del diario, el uso de específicas técnicas y géneros en la producción discursiva apuntarían a instalar una cuasi ontología profesional. Así, el periodista sería concebido como un simple "testigo de la historia". La información debía ser escrita en forma breve, precisa y escueta, sin adjetivos ni opiniones. Es el propio carácter de este periodismo de nuevo tipo y sus técnicas consiguientes lo que aseguraría su neutralidad: “...Esta nueva modalidad demostró que las noticias podían hablar por si mismas, sin el comentario y la interpretación del cronista" (CHARNLEY, 1971: 229).

Con ello, a nivel de los géneros se consolida lo ya dicho, en cuanto al predominio de aquellos que garantizan la subordinación de sujetos individuales de enunciación por otros que surgen de moldes escriturales desde donde se expresa el periódico en tanto actor social y/o político. Junto a los procesos ya mencionados acerca de la diversificación del carácter y tipo de los periódicos y diarios, primero, de toda la prensa escrita y de los medios de comunicación en general, después, por un lado y de la configuración de públicos diversos y especializados, vinculados a espacios urbanos y circuitos culturales ampliados y nuevos, por otro, cabe detenerse en otro plano de transformaciones, estrictamente relacionado con las prácticas y rutinas propias de la lógica profesional del periodismo.

\section{Géneros periodísticos y prensa liberal moderna}

Desarrollados al calor y como producto de la transformación que vive la prensa occidental desde mediados del siglo XIX, los géneros -entendidos como herederos de los géneros literarios- satisfacen en la actividad periodística una necesidad inmediata y urgente, cual es la de facilitar el trabajo colectivo, todo lo cual está a la base del surgimiento de un profesional determinado, diestro en el manejo de técnicas de selección y producción de informaciones. El formato aparece no sólo como instrumento específico de construcción textual estandarizada, sino también, entre otras funciones, como mecanismo impulsor de la división del trabajo intelectual dentro del diario y la especialización profesional. La empresa periodística, como estructura productiva moderna, 
requería de los géneros como moldes básicos, totalmente impersonalizados, en los cuales volcar la materia prima: los hechos convertidos en acontecimientos y éstos en noticias (RodRIGO Alsina, 1992).

Lo cierto en el caso de la prensa moderna es que el género vino a satisfacer una necesidad estrictamente productiva. Si el negocio es vender información cualificada como noticia, la rapidez y la inmediatez productiva debían adecuarse al carácter efímero y volátil del producto. La plena consolidación de los géneros periodísticos, así como el desarrollo de una estructura colectiva de producción de noticias debió ser producto de un paulatino proceso que, en el caso de nuestro país, es posible advertir ya desde mediados del siglo XIX en una dinámica compleja de relaciones entre el campo literario y el periodístico propiamente tal en formación.

En ese marco, emerge un género particular, por así decirlo a medio camino entre lo literario y lo periodístico, terreno híbrido que le permitía a los literatos aceptar la condición de asalariados de las nacientes empresas periodísticas, a través del llamado proceso de "autonomía-delegación" (CATALÁN, 1985). Si bien, el autor se refiere particularmente a las relaciones entre las hegemonías sociales y el campo literario, es también posible usarlo para entender cómo solucionar la paradoja entre la posición ideológica del periódico o revista y la distancia "neutral" frente a los hechos en que la naciente empresa periodística coloca al periodista o cronista. Para CATALÁN, la relativa autonomización de la literatura (y el periodismo, agregamos) encuentra su correlato en la reformulación de los vínculos hegemónicos del sistema cultural dominante con los productores literarios, a través de lo que llama "delegación, en efecto, porque si bien en ella el nuevo estamento de escritores pasa a asumir la producción misma de los bienes literarios, los grupos socialmente hegemónicos se reservan o implementan mecanismos que permiten controlar en algún grado el sentido de esa producción" (CATALÁN, 1985: 73).

Por otra parte, vista desde la reflexión y la práctica actuales de la prensa moderna, la crónica periodística aparece como uno de los géneros más difícilmente clasificable. Sin embargo, su vigencia (aunque relegada a planos secundarios por otros géneros como el informativo y el reportaje interpretativo) obliga a que diversos autores hayan intentado definirla (MARTín VIVALDI, 1993). Este autor asume como punto de partida una cierta ambiguedad, que de alguna forma incorpora la maleabilidad del género, estableciendo que se trata de un género informativo, pero que es algo más que pura información y algo más que un reportaje interpretativo. Un elemento distintivo de la crónica sería justamente la interpretación o valoración de los hechos que en ella se narran: “...El cronista, al relatar algo, nos da su versión del suceso; pone en su narración un tinte personal. No es la cámara fotográfica que reproduce un paisaje; es el pincel del pintor que interpreta la naturaleza, prestándole un acusado matiz subjetivo" (MARTíN VIVALDI, 1993: 128).

El otro elemento constituyente fundamental son los hechos, textualmente producidos como noticia; sin ellos, la crónica dejaría de ser periodística. Martin Vivaldi en el texto antes citado agrega que no existe un estilo objetivo pre-determinado para la crónica y que el cronista está libre de los modelos formales, cuestión justamente contraria a la que ocurriría a lo largo del siglo XX con géneros como el párrafo informa- 
tivo (CHARNLEY, 1971) o el reportaje (SANTIBÁÑEz, 1974), ambos sometidos a estrictos cánones de producción escritural, justamente en la búsqueda de la impersonalidad del texto, asegurando que el sujeto de la enunciación sea el diario como estructura.

En el caso de la crónica periodística la libertad estilística tendría un sólo límite fundamental establecido por el carácter noticioso del hecho en torno al cual se escribe, ya que como vimos, allí estaría la clave específica de su identidad, en tanto forma periodística y no literaria. Este límite, sin embargo, no se restringe al plano del contenido, sino que se manifiesta con similar importancia en el plano expresivo, el cual debe someterse a las exigencias comunicativas, a la presencia heterogénea del lector, en sentido amplio. Así, el estilo de la crónica ha de ser claro, sencillo, conciso; revelador de un contenido objetivo que se comunica a alguien. En ese marco, valen todos los recursos estilísticos y retóricos: comparación, metáfora, ironía, paradoja e incluso, aunque mesuradamente, la hipérbole. La única exigencia que fluye del marco es la claridad comunicativa en función de un público determinado. En definitiva, toda crónica tiene un sentido y entraña una significación: se escribe de algo y por algo o para algo; se cuenta un hecho significativo y se le da al relato un sentido estimativo-axiológico; se narra un suceso y se procura describir su valor.

Dicho proceso se extendió por toda América Latina y ha sido objeto de numerosos estudios (RotKer, 2005), lo que llevó a Julio RAmos (1989: 85) a afirmar acerca de los literatos que incursionaron en los periódicos, usando la crónica como campo predilecto, que: "Muchos de ellos provenientes de las nuevas clases medias, sin un 'capital simbólico' (o efectivo) garantizado por filiación oligárquica, los escritores finiseculares $[\ldots]$ que defendían la alternativa del mercado y la profesionalización, se situaban en contra de la zona más reaccionaria del campo, que manejaba aún un concepto civil de la Literatura [...] Por supuesto, también Martí, Gutiérrez Nájera, o luego Darío, se distanciarían de la otra posición clave en el campo finisecular: la literatura propiamente 'industrial', que muchos literatos relacionarían con la emergencia de un nuevo tipo de periodista, escritor de noticias y folletines".

En un comienzo este tipo de textos fue más cercano al cuadro de costumbres inglés o francés o a la chronique periodísticafrancesa, especialmente elfait divers, de Le Figaro, de París, según afirma RotKer (1992). Para el caso chileno, Ossandón recuerda las que llama coloraciones locales de José Joaquín Vallejo (Jotabeche), Domingo Faustino Sarmiento o Vicente Pérez Rosales. Hacia finales del siglo, continúa, ya se encontraba constituido un corpus con peso literario y periodístico y una generación de cronistas en actividad. Algo a destacar en ello es que buena parte de sus componentes son cada vez más periodistas que literatos. Entre esos nuevos profesionales de la información, en el caso chileno, OsSANDón destaca a los hermanos Arteaga Alemparte, en especial Justo y añade que se trataba, en muchos casos, de jóvenes que se habían incorporado a los periódicos y en su práctica profesional se habían convertido en cronistas; en algunos de ellos, como es el caso de Díaz Garcés y Silva Vildósola, luego de algunos años y desde el periodismo incursionarían en la literatura (MundY, 1996).

El ámbito de la crónica se constituye en el período analizado, en un espacio de emergencia de un nuevo tipo de discurso y producción intelectual, al decir de RAMOS 
(1989) con características de "...vitrina de la vida moderna". Ahora bien, en la dirección señalada es que cabe resaltar las crónicas de José Martí. La citada Susana RoTKER hace notar que más de la mitad de la obra del cubano se encuentra en periódicos y que entre 1880 y 1892 escribió más de 400 crónicas reproducidas en diversos diarios latinoamericanos como La Nación, de Buenos Aires; La Opinión Nacional, de Caracas; La Opinión Pública, de Montevideo; La República, de Tegucigalpa; El Partido Liberal, de México y La América, de Nueva York (1992). Por su parte, y aunque la autora no lo menciona, no es menor la presencia de crónicas martianas en la prensa chilena. Una exhaustiva investigación realizada al respecto consigna que Martí publicó un total de 70 artículos en la prensa chilena entre 1881 y 1895. De ellos, 44 aparecieron en La Epoca; 15 en El Mercurio de Valparaíso; 6 en La Libertad Electoral: 4 en El Ferrocarril y uno en El Sur de Concepción (BENíTEZ, 1995).

Si bien hay una serie de elementos que ubican a Martí cercano o al interior de los modernistas, y donde la mayor parte de los estudios lo ha ubicado, también hay otros no menos importantes que lo alejan. El impacto de la modernización capitalista de sello oligarca que hemos mencionado al comienzo de este texto, generó efectivamente la sensación de caos y vacío existencial, especialmente en ciertos sectores intelectuales, muchas veces arrojados a subsistir en el incipiente mercado cultural. Así, la bohemia de los cafés, los ateneos, las redacciones y las tertulias fueron el lugar de emergenciade grupos de literatos e intelectuales críticos frente a un mundo que parecía entregado al imperio del dinero en circulación, ahora visto en forma descarnada. Sin embargo, como señala José Luis Romero (2001: 290) "sus miembros estaban dentro de algunas de las varias direcciones que apuntaban en el seno de las nuevas burguesías".

A contrapelo de los trabajos citados de RAMOs, ROTKER y otros, José Luis ROMERO no valora en los modernistas ningún contenido crítico o rupturista al orden capitalista que se estaba consolidando: "el modernismo de los poetas -el mejicano Gutiérrez Nájera, el cubano Julián del Casal, el uruguayo Julio Herrera y Reisig, el argentino Leopoldo Lugones y sobre todo el nicaragüense Rubén Darío- recogía y expresaba la sensibilidad de los exquisitos; pero de los poderosos exquisitos, a quienes seducía el mundo refinado del lujo y, a veces, el refinado lujo del poder. Más que disconformismo había en él un rechazo de lavulgaridad, que se confundía fácilmente con el apresurado aristocratismo de las nuevas burguesías" (ROMERO, 2001: 290).

No nos parece casual que la cita no incluya a Martí compartiendo ese tipo de modernismo de los poetas. Si hay algo que lo distingue de esos grupos es el compromiso político de Martí con la causa de la independencia nacional deCuba y con la perspectiva que abre de sentar las bases de un proyecto cultural e identitario para América Latina. En ese sentido, en Martí nos aparecen, al menos, tres facetas: el literato, el periodista y el político, aquel que, mirando las convulsiones y crisis del contexto contradictorio que vivió América Latina en el período, es capaz de colocar una mirada más allá, pero a la vez desde la experiencia cotidiana de esos fenómenos, al señalar: "Asístese como a una descentralización de la inteligencia. Ha entrado a ser lo bello dominio de todos". 


\section{Conclusiones}

El proceso de modernización capitalista que se vive en las últimas décadas del siglo XIX en América Latina y en Chile (Romero, 2001) provocó que la tendencia profunda de transformación del quehacer periodístico se fuera paulatinamente imponiendo. Lo anterior significó, entonces, una transición entre un tipo de periodismo, más ligado a la vocería y difusión de doctrinas como expresión de partidos o grupos políticamente definidos, a otro, más bien ligado a las exigencias de un mercado informativo en creciente desarrollo y expresadas fundamentalmente bajo la forma de la ampliación del círculo de lectores y la captura de publicidad.

Ello se expresó en diversos planos. Junto a los procesos de diversificación del carácter y tipo de los periódicos y diarios, primero, de toda la prensa escrita y de los medios de comunicación en general, después, por un lado y de la configuración de públicos diversos y especializados, vinculados a espacios urbanos y circuitos culturales ampliados y nuevos, por otro, cabe recalcar otro plano de transformaciones, estrictamente relacionado con las prácticas y rutinas propias de la lógica profesional del periodismo. Nos referimos a la progresiva aparición de los géneros periodísticos, como forma de producción textual específica que requería de un profesional ad-hoc: el reportero o periodista.

De este modo, estaban creadas las condiciones necesarias para el desarrollo que la prensa latinoamericana y chilena vivió durante el siglo XX, con sus conflictos, tensiones, grandezas y miserias, pero como diría el sentido común de la profesión, eso ya es otra historia.

\section{Referencias bibliográficas}

AGUIRRE, Carlos (2004): "Los irrecusables datos de la estadística del crimen: la constancia social del delito en la Lima de mediados del siglo XIX", en ALONSO, Paula (compiladora): Construcciones impresas. Panfletos, diarios y revistas en la formación de los estados nacionales en AméricaLatina, 1820-1920, Buenos Aires, Fondo Cultura Económica, Pp. 273-295.

ÁLVAREZ, Jesús y MARTÍNEZ, Ascensión (1992): Historia de la prensa hispanoamericana. Madrid, Mapfre.

BARRERA, Carlos (2004): Historia del periodismo universal. Barcelona, Ariel Comunicación.

BARROS ARANA, Diego (2003): Un decenio de la historia de Chile (1841-1851). Santiago de Chile, Instituto de Historia, P. Universidad Católica de Chile.

BENÍTEZ, Jorge (1995): José Martí y Chile. Santiago de Chile, LOM Ediciones.

CATALÁN, Gonzalo (1985): "Antecedentes sobre la transformación del campo literario en Chile entre 1890 y 1920", en CATALÁN, Gonzalo y BRUNNER, José Joaquín: Cinco Estudios Sobre Cultura y Sociedad. Santiago de Chile, FLACSO, Pp. 69-175.

CHARNLEY, Mitchell (1971): Periodismo Informativo. Buenos Aires, Troquel. MARTÍN VIVALDI, Gonzalo (1993): Géneros periodisticos. Madrid, Paraninfo. 
MUNDY, Evangeline (1996): Joaquín Díaz Garcés. Su vida y su obra (1877-1921). Santiago de Chile, El Mercurio S.A.P.

OSSANDÓN, Carlos (1998): El crepúsculo de los sabios y la irrupción de los publicistas. Santiago de Chile, Arcis-LOM.

OSSANDÓN, Carlos y SANTA CRUZ A., Eduardo (2001): Entre las alas y el plomo. La gestación de la prensa moderna en Chile. Santiago de Chile, Arcis-LOM.

POSADA, Eduardo (2004): “¿Libertad, libertinaje, tiranía?. La prensa bajo el Olimpo Radical en Colombia, 1863-1885", en ALONSO, Paula (compiladora): Construcciones impresas. Panfletos, diarios y revistas en la formación de los estados nacionales en AméricaLatina, 1820-1920. Buenos Aires, Fondo Cultura Económica, Pp. 183-201.

RAMOS, Julio (1989): Desencuentros de la Modernidad en América Latina. Literatura y política en el siglo XIX. México, Fondo de Cultura Económica.

RODRIGO ALSINA, Miquel (1992): La construcción de la noticia. Barcelona, Paidós Comunicaciones.

RODRÍGUEZ MENDOZA, Emilio (1955): Alfredo Irarrázaval Zañartu. Adición a ¡Como si fuera ayer!. Santiago de Chile, Editorial Jurídica de Chile.

ROMERO, José Luis (2001): Latinoamérica. Las ciudades y las ideas. Buenos Aires, Siglo XXI.

ROTKER, Susana (1992): Fundación de una escritura. Las crónicas de José Martí. La Habana, Ediciones Casa de las Américas

ROTKER, Susana (2005): La invención de la crónica. México, Fondo Cultura Económica.

SANTA CRUZ ACHURRA, Eduardo (2009): “Cuestión de patricios y letrados. Prensa y espacio público en América Latina y Chile (1820-1850)", en Mapocho, $\mathrm{n}^{\mathrm{o}} 66$. Santiago de Chile, Segundo Semestre 2009, DIBAM, Proyecto Fondecyt N ${ }^{\mathrm{o}}$ 1085029.

SANTIBÁÑEZ, Abraham (1974): Periodismo Interpretativo. Santiago de Chile, Editorial Andrés Bello.

SILVA CASTRO, Raúl (1958): Prensa y periodismo en Chile. Santiago de Chile, Ediciones Universidad de Chile.

URIBE ECHEVARRÍA, Juan (1973): Tipos y cuadros de costumbres en la poesía popular del siglo XIX. Santiago de Chile, Pineda Libros.

VALDEBENITO, Alfonso (195 ): Historia del periodismo chileno. Santiago de Chile, Círculo de Periodistas de Santiago.

VÁZQUEZ MONTALBAN, Manuel (1985): Historia y Comunicación Social. Madrid, Alianza.

VIAL CORREA, Gonzalo (1983): Historia de Chile (1891-1973). La sociedad chilena en el cambio de siglo. vol.1. Santiago de Chile, Santillana del Pacífico. 
WEILL, Georges (1994): El periódico. Orígenes, evolución y función de la prensa periódica. México, UTEHA, Noriega Editores. 